\title{
CARACTERIZAÇÃO FUNCIONAL DA MÍMICA FACIAL NA PARALISIA FACIAL EM TRAUMA DE FACE: RELATO DE CASO CLÍNICO
}

\author{
Functional characterization of facial mimicry in facial paralysis \\ of face trauma: a clinical case report
}

Leila Bonfim de Jesus ${ }^{(1)}$, Daniele Fontes Ferreira Bernardes ${ }^{(2)}$

\begin{abstract}
RESUMO
Tema: mímica facial na paralisia facial ocasionada por trauma de face. Procedimentos: estudo de caso da mímica facial de um paciente com paralisia facial periférica no lado direito com sequelas decorrente de trauma por projétil de arma de fogo, adulto jovem do sexo masculino, comerciante autônomo. Foi realizada análise documental e fotográfica por meio de anamnese, avaliação e escala de graduação de da paralisia facial House e Brackmann. Resultados: na avaliação da paralisia facial em repouso encontrou-se no lado direito (lesado): desvio de comissura labial, desvio de filtro, narina mais elevada e olho mais aberto. Em movimento, ainda no lado da lesão, observou-se: abolição de rugas frontais, incompetência no fechamento ocular natural e fechamento completo ao forçar, ausência de elevação de narina, rima naso-labial mais pronunciada, protrusão labial desviada para este lado, pouca retração labial, eversão de lábio inferior, comissura labial elevada, desvio de filtro, reduzida capacidade de inflar bochechas. O paciente apresentou sincinesia de olho/lábios e contratura com hipertonia de frontal, prócero, levantador da asa do nariz, risório, zigomático maior, zigomático menor, levantador do lábio superior, depressor do lábio inferior, mentual no lado da lesão e a fratura ocorreu em côndilo direito e o paciente referiu dor orofacial ao dormir e ao mastigar no lado comprometido. Conclusão: a lesão do nervo facial associada ao trauma de face provocou a alteração da mímica facial no lado direito o que gerou desfiguramento e distúrbios da mastigação.
\end{abstract}

DESCRITORES: Nervo Facial; Paralisia Facial; Músculos Faciais; Ferimentos por Arma de Fogo

\section{INTRODUÇÃO}

A mímica é a arte de imitar, de exprimir os pensamentos por meio dos gestos e da expressão fisionômica 1. A mímica facial é uma ferramenta substancial utilizada na comunicação humana, já que por meio dela é possível identificar sentimentos e idéias. A mobilidade dos músculos da boca é imprescindível para a fala, expressão e também alimentação do indivíduo, motivos pelos quais o

(1) Fonoaudióloga Graduada pelo Centro Universitário Jorge Amado - UNIJORGE; Especializanda em Motricidade Orofacial pela União Metropolitana de Ensino -UNIME.

(2) Docente da União Metropolitana de Educação e Cultura UNIME; Mestre pela Faculdade de Medicina da Universidade de São Paulo - FMUSP.

Conflito de interesses: inexistente paciente que apresenta paralisia facial procura o atendimento fonoaudiológico ${ }^{2}$. O controle preciso da musculatura facial permite variações sutis na fisiologia muscular, necessárias para as funções referidas acima ${ }^{3}$.

A paralisia facial é uma situação de impacto, na qual a pessoa perde a possibilidade da comunicação não-verbal, ou seja, das informações fornecidas ao interlocutor que apenas as palavras expressas oralmente não são capazes de transmitir ${ }^{4}$.

Entre as diversas causas de Paralisia Facial Periférica (PFP), a etiologia traumática assume grande importância nos dias atuais. Após a paralisia de Bell, os casos traumáticos são a segunda causa de PFP. Entre as causas de PFP traumática encontram-se as decorrentes de: trauma craniocerebral (TCC) com maior número; lesões iatrogênicas do 
nervo facial; lesões por projétil de arma de fogo; ferimentos cortantes da face e lesão do nervo facial no parto ${ }^{5}$.

Os traumas de face vêm representando um campo de preocupação fonoaudiológica, uma vez que interferem no desempenho do sistema estomatognático e, consequentemente, na eficiência das funções realizadas ${ }^{6}$.

A PFP em fase de sequelas caracteriza-se por presença de rugas na testa no lado afetado, olhos mais estreitos ou contraídos do lado comprometido, rima naso-labial mais pronunciada do lado comprometido, filtro labial desviado para o lado da lesão e comissura labial elevada do lado afetado evidenciando a presença de contraturas ${ }^{7}$.

As principais alterações no sistema estomatognático geram comprometimento na função mastigatória, alteração na preensão dos lábios, diminuição da pressão intra-oral, alteração da deglutição, em alguns casos, redução da sensibilidade gustativa dos dois terços anteriores da língua e instabilidade na articulação da fala e da expressão facial ${ }^{8}$.

O presente artigo tem por finalidade descrever, de acordo com a avaliação fonoaudiológica, as características da mímica facial na paralisia facial causada por trauma de face.

\section{APRESENTAÇÃO DO CASO CLÍNICO}

Este estudo baseia-se no relato de caso de um adulto jovem de 28 anos, do gênero masculino, solteiro, trabalhador autônomo que realizou atendimento fonoaudiológico em uma clínica-escola. Ele apresenta PFP do lado direito da face em decorrência de acidente por arma de fogo. A queixa do paciente foi: "não consigo mastigar e mexer o lado direito do meu rosto. Meu olho direito ficou com o movimento lento."

Para elaboração deste trabalho, foi realizada análise documental dos dados do prontuário referentes à anamnese, avaliação e relatórios terapêuticos fonoaudiológicos, além de exames e avaliações multidisciplinares.

A anamnese e avaliação fonoaudiológica permitiram a obtenção de dados sobre origem da paralisia facial periférica, seu início, sintomas, tratamentos anteriores e graduação da mobilidade e tonicidade dos músculos da mímica facial.

Este estudo foi autorizado mediante assinatura do Termo de Consentimento Livre e Esclarecido elaborado para fins específicos desta pesquisa, segundo a Resolução no. 196/96 e teve aprovação do Comitê de Ética em Pesquisa (CEP) do Hospital Espanhol - Salvador - Bahia, processo no. 034/2010.

\section{Dados da anamnese}

A anamnese foi realizada com base em protocolo de Chevalier ${ }^{9}$.

O paciente foi vítima de trauma de face e cervical, no lado direito, por arma de fogo há três anos. Constatou-se um trajeto de projétil de arma de fogo, extra-craniano, em nível de nervo petroso superficial maior. $O$ primeiro entrou pela cavidade oral, lesou côndilo direito e se alojou na articulação em região cervical Zona II (estende-se da cartilagem cricóide até o ângulo da mandíbula), causando uma paralisia facial periférica ipsilateral. A paralisia facial periférica foi súbita e apresentou sintomas no lado lesado, do tipo: incompetência para fechar olho, ressecamento ocular, dores de ouvido, hipoacusia, bochecha caída, dificuldade para mastigar e sensibilidade gustativa alterada.

Ao ser atingido, o mesmo foi socorrido e levado para um hospital especializado em atendimento de emergência, no qual foi atendido e encaminhado para realizar o exame de tomografia computadorizada de crânio, que não evidenciou alterações. Foi submetido ainda à antibioticoterapia, sintomatologia e profilaxia do tétano. No dia seguinte, o paciente teve alta e após dois dias do acidente passou por cirurgia para remoção de projétil e teve alta hospitalar no mesmo dia. Segundo o paciente esta fatalidade tornou sua vida um caos devido à mudança da estética facial, dificuldade para mastigar e dor orofacial no lado direito.

\section{Dados da avaliação}

A avaliação foi realizada com base em protocolo de Chevalier ${ }^{9}$ e a graduação da paralisia foi estabelecida de acordo com a Escala House-Brackmann ${ }^{10}$. Para avaliação em repouso foi registrada, com câmera digital, a face em posição habitual. Foram solicitados comandos verbais e visuais, para avaliação em movimento, considerando cada músculo da mímica facial individualmente e classificando-os como normal, alteração parcial ou total. O paciente devia seguir tais comandos: fazer "cara de assustado", "cara de bravo", "cara de cheiro ruim", "raspar bigode", "fechar os olhos suavemente", fechar os olhos com força, "sorriso fechado", "sorriso aberto", comprimir bochecha, mostrar dentes inferiores, empurrar queixo para cima, contrair músculo do pescoço e "fazer bico". Realizou-se a classificação e graduação dos itens: mobilidade da musculatura da mímica facial, fechamento ocular, avaliação do tônus em repouso, na contratura e avaliação das sincinesias.

Como o presente estudo se baseou em imagens da mímica facial, os resultados serão apresentados de forma descritiva. 


\section{RESULTADOS}

Ao analisar a mímica facial a partir da observação visual presencial e dos registros fotográficos pôde-se observar que: em repouso o paciente apresentou no lado direito (lesado): desvio de comissura labial, desvio de filtro labial, sulco nasolabial acentuado, narina mais elevada e olho mais aberto (figura 1). Na Escala House-Brackmann o paciente alcançou o grau III. A mobilidade dos principais grupos musculares da mímica facial foi classificada como grau II. Em movimento, ainda no lado da lesão, observou-se: abolição de rugas frontais (figura 2), incompetência no fechamento ocular natural com grau II e fechamento completo no forçado, ausência de elevação de narina, rima naso-labial mais pronunciada, protrusão labial desviada para este lado (figura 3), limitação da retração labial, eversão de lábio inferior, comissura labial elevada, desvio de filtro, reduzida capacidade de inflar bochechas.

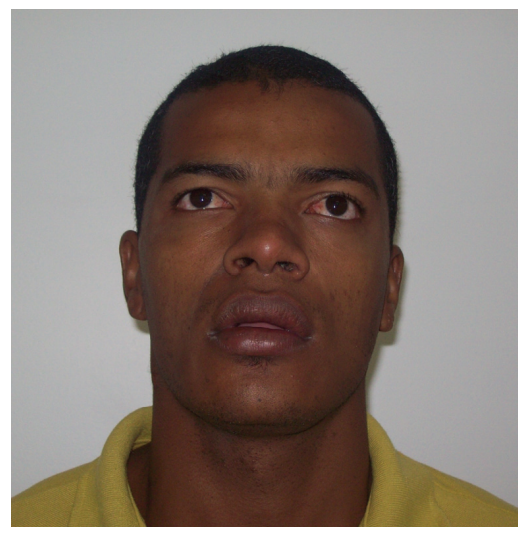

Figura 1 - Face em repouso: sulco nasolabial acentuado à direita, narina direita levemente elevada, pálpebra inferior esquerda caída

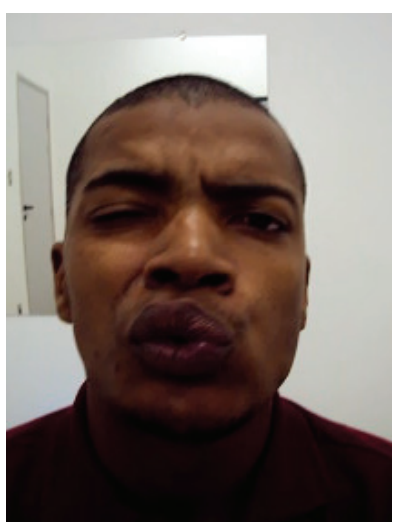

Figura 2 - Ausência de rugas frontais no movimento "cara de assustado" e presença de sincinesia de retração labial e oclusão ocular à D
Na prova de avaliação das sincinesias foi solicitado que o paciente fizesse "sorriso aberto" e verificou-se grau II de sincinesia de olho/lábios (figura 4) apresentada não só neste movimento como em todos os comandos para movimentos labiais. Ao observar o tônus da musculatura em repouso foi possível identificar a presença de contratura com hipertonia no lado da lesão. $\mathrm{Na}$ avaliação das funções estomatognáticas encontrou-se apenas alterações da mastigação, com predominância unilateral do lado esquerdo e realização de contrações exageradas dos músculos mastigatórios e do platisma para conseguir mastigar do lado direito. A queixa principal do mesmo foi a dificuldade em aceitar seu padrão facial após paralisia facial periférica e a baixa auto-estima. Seria interessante para o estudo a realização do exame de eletromiografia de superfície para confirmar em valores o grau de participação da musculatura da mímica facial, porém o paciente não deu continuidade ao tratamento.

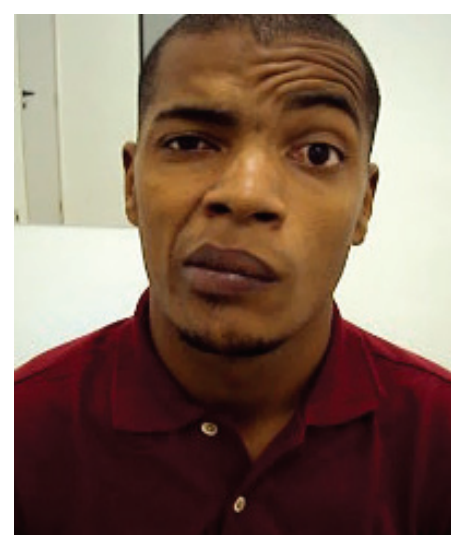

Figura 3 - Desvio de protrusão labial para lado lesado e presença de sincinesia de oclusão ocular à $\mathrm{D}$

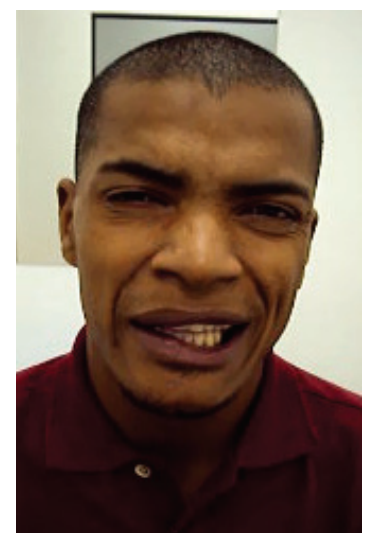

Figura 4 - Sincinesia de olho-boca no "sorriso aberto". 


\section{DISCUSSÃO}

Segundo Madeira², os músculos da expressão facial são orbicular da boca, levantador do lábio superior, levantador do lábio superior e da asa do nariz, zigomático menor, levantador do ângulo da boca, zigomático maior, risório, bucinador, depressor do ângulo da boca, depressor do lábio inferior, mentoniano, platisma, orbicular do olho, occipitofrontal, prócero, corrugador do supercílio e nasal; e suas contrações produzem na face variações na forma de orifícios anatômicos, pregas e sulcos, que alteram a fisionomia e exteriorizam os sentimentos das pessoas ${ }^{11}$. Lianza e Nogueira (2006) ${ }^{12}$ descreveram, que os músculos faciais são espirais e diagonais e estão topograficamente dispostos para movimentos simétricos. A contração forte dos músculos circulares ao redor da boca e dos olhos requer reações de estiramento e encurtamento de outros grupos faciais, inclusive os da cabeça. Na paralisia facial esse equilíbrio é prejudicado, devido comprometimento do nervo facial, ocorrendo alterações da mímica facial.

A função primária do nervo facial é a inervação motora dos músculos da expressão facial. É composto por duas raízes distintas: o nervo facial próprio (a raiz motora) e o nervo intermédio (a raiz sensitiva). As raízes emergem do tronco encefálico na borda inferior da ponte, lateralmente à extremidade superior da oliva bulbar, entram no meato acústico interno, seguem para parte petrosa do osso temporal. O nervo facial cursa, então dentro de um canal ósseo e sai do crânio pelo forame estilomastóideo. O nervo, agora em sua localização extratemporal, entra na glândula parótida e começa a se dividir em ramos têmporo-facial e cervicofacial, terminando como uma verdadeira rede na musculatura da face que atua na expressão facial ${ }^{13}$.

As características clínicas encontradas em nossa pesquisa indicaram uma paralisia facial periférica traumática, em fase de sequelas, em adulto jovem de 28 anos com início súbito. O paciente foi acidentado em maio de 2008 e após 3 anos de paralisia facial periférica no lado direito, encontravase em fase de seqüela.

Segundo estudo realizado, a PF traumática é a segunda causa mais frequente de PFP, ficando atrás somente dos casos ditos idiopáticos ${ }^{14}$. De acordo com a literatura, há maior ocorrência no sexo masculino, com predomínio de casos súbitos. O trauma pode ocorrer como conseqüência tanto de fratura de face como crânio-encefálicas ${ }^{15}$.

Quanto à faixa etária do paciente, esta confirma os achados de uma pesquisa que observou que os traumas de face ocorrem frequentemente na faixa de 20 a 40 anos $^{16}$.
O paciente apresentou uma fratura condilar, com queixa de dor orofacial à direita e mastigação lenta. Comprometimentos semelhantes foram observados em pesquisa na qual em casos de fraturas de côndilo, os pacientes apresentaram sintomas como: dor na musculatura facial, cansaço e redução da força ao mastigar, limitação e desvios dos movimentos mandibulares ${ }^{17}$.

No início da fase flácida o paciente apresentou redução do lacrimejamento no olho direito, que persistiu até a fase de seqüelas, juntamente com a dificuldade para fechar o olho no fechamento natural.

Também foram descritos casos de paralisia facial periférica onde se observou diminuição do lacrimejamento do lado acometido na fase flácida em paralisia facial de origem neoplásica ${ }^{18}$.

Para reabilitação na paralisia facial deve-se ter em mente as duas fases: flácida e de sequelas. Conhecimentos das especificidades da musculatura orofacial são de importância fundamental ${ }^{19}$.

O impacto da condição facial na vida do paciente parece não depender do grau das sequelas, ou seja, o grau de incômodo com relação à sincinesia e/ou contratura, parece relacionar-se mais a fatores individuais do que ao grau obtido na avaliação clínica ${ }^{20}$.

O grau de recuperação da paralisia facial depende do tipo de lesão, duração do período de desnervação, conexões motoras e sensoriais (direcionamento de fibras), grau de reinervação e estado do músculo. Ou seja, a estimulação da saúde do músculo é imprescindível para um bom resultado, porém, não é responsável isolado pela recuperação completa ${ }^{21}$.

A demora para o início da terapêutica piora muito o prognóstico. Bogar, Calda Neto, Marone e Miniti 22 salientam que é importante intervir nos casos de paralisia facial traumática, mesmo passados meses, pois a maioria dos pacientes apresenta melhora. Bernardes, Goffi Goméz e Bento ${ }^{23}$ demonstraram em seu artigo que o resultado do tratamento miofuncional é melhor quando o paciente inicia o tratamento até trinta dias após a lesão nervo facial.

O diagnóstico pode ser complementado pela a utilização da eletromiografia de superfície, que se tornou uma ferramenta aliada à Motricidade Orofacial. Este exame tem sido importante nas terapias miofuncionais orofaciais por ser um método objetivo e quantificador, que mensura os potenciais elétricos emanados pelos músculos no momento da contração muscular ${ }^{24}$.

Em estudo realizado por Bernardes, Goffi Goméz e Bento ${ }^{25}$, no qual foram avaliados os valores da atividade eletromiográfica dos músculos frontal, orbicular dos olhos, orbicular da boca em indivíduos normais e pacientes portadores de 
paralisia facial periférica foi encontrado que em indivíduos normais a média dos potenciais eletromiográficos para ambos os lados da face é semeIhante, demonstrando que a integridade do nervo facial é fundamental para o equilíbrio da mímica facial. Em indivíduos com paralisia facial periférica na fase de sequela observou-se a média dentro da normalidade na elevação da testa e fechamento ocular, porém com índice alto neste movimento para o segmento lábio, evidenciando a sincinesia olho/lábio.

\section{CONCLUSÃO}

De acordo com o estudo podemos concluir que a paralisia facial periférica por trauma de face causou sequelas como a hipertonia da musculatura lesada e sincinesias de lábios e olhos na hemiface direita, de modo que comprometeu a realização de gestos como o sorriso e expressões de alegria ou tristeza e dificultou a mastigação no lado lesado.

\begin{abstract}
Background: facial mimicry in the paralysis of the face that occurs because of a trauma in the face. Procedures: case study of facial mimicry of a patient with peripheral facial paralysis, on the right side of the face, with sequels that happened because of a trauma because of a fire gun projectile; a young man, a self-employed trader. A documental and photographical analysis was held through the anamnesis and graduation scale of House and Brackmann's facial paralysis. Results: in the evaluation of the facial paralysis, in a resting state, we found on the right side (the injured one): diversion of lip commissure, diversion of the filter, more elevated nostril and more open eye. In movement, yet on the side of the injury, it was observed: elimination of frontal wrinkles, incompetence in the ocular closure and in the complete closure, absence of elevation of the nostril, a more pronounced nasolip rhyme, lip protrusion diverged to this side, little lip retraction, destruction of the inferior lip, elevated lip commissure, diversion of the filter, reduced capacity of inflating the cheeks. The patient presented synkinesia of eyes / lips and contraction with hypertonia of frontal, procerus, lifter of the nose's wing, risorius, higher zygomatic, lower zygomatic, lifter of superior lip, depressive of inferior lip, mentalis on the side of the lesion and the fracture happened on the right condyle and the patient reported orofacial pain when sleeping and chewing on the injured side. Conclusion: the lesion of the facial nerve that was associated with the face trauma provoked the alteration of the facial mimicry on the right side and generated disfiguration and disturbances in the chewing act.
\end{abstract}

KEYWORDS: Facial Nerve; Facial Paralysis; Facial Muscles; Injuries caused by Fire Guns

\section{REFERÊNCIAS}

\section{Koogan A, Houaiss A. Enciclopédia e Dicionário} llustrado. Rio de Janeiro: Delta, 1997.

2. Madeira MC. Anatomia da face. São Paulo: Sarvier, 1998.

3.Calais LL, Goffi-Gomez MVS, Bento RF, Comerlatti LR. Avaliação funcional da mímica na paralisia facial central por acidente cerebrovascular. Pró-Fono. 2005; 17(2): 213-22.

4. Bernardes DF, Gomez MV, Pirana S, Bento RF. Functional profile in patients with facial paralysis treated in the myofunctional approach. Pró- Fono. 2004; 16(2):151-8.
5. Lazarini PR. Tratamento da paralisia facial periférica pós-trauma craniocerebral, ACTA ORL. 2005; 23(3):6-13.

6. Bianchini ME. Traumas de face: atuação fonoaudiológica, caracterização, proposta terapêutica e resultados In: Comitê de Motricidade Orofacial da SBFa. Motricidade orofacial: como atuam os especialistas. São Paulo: Pulso; 2004. p. 107-14.

7. Bernardes DFF. A Contribuição da análise eletromiográfica de superfície para a definição da fase de evolução da paralisia facial periférica: fase flácida ou fase de sequelas [dissertação de mestrado]. São Paulo (SP): Faculdade de Medicina, Universidade de São Paulo; 2008.

8. Brach JS, Van Sweringen J, Delitto A, Johnson PC. Impairment and disability in patients with facial 
neuromuscular dysfunction. Otolariyngol Head Neck Surg. 1997;117(4):315-21.

9. Chevalier MA et al. Avaliação da função motora da face nas lesões periféricas e centrais. In: Lacôte M, Chevalier AM, Miranda A, Bleton JP, Stevenin P. Avaliação clínica da função muscular. São Paulo: Manole, 1987: 13-35.

10. House JW, Brackmann DE. Facial nerve grading system. Otolgol. Head and Neck Surg. 1985; 93:146-7.

11. Madeira MC. Anatomia da face. São Paulo: Sarvier, 1998: 1-77.

12. Lianza S, Nogueira MARJ. Tratamento médico da paralisia facial periférica na visão do fisiatra. In: Lazarini PR, Fouquet ML. Paralisia facial: avaliação, tratamento e reabilitação. São Paulo: Lovise, 2006: 131-8.

13. Fouquet $\mathrm{ML}$, Lazarini PR. Atuação fonoaudiológica na paralisia facial periférica. In: Lopes Filho O. Tratado de fonoaudiologia. São Paulo: Tecmedd, 2004: 971-84.

14. Vasconcelos BEC; Dias E, Dantas WRM, Barros ES e Monteiro GQM - Paralisia facial periférica traumática. Rev. Cir. Traumat. Buco - MaxiloFacial. 2001; 1 (2): 13-20.

15. Pinna BR, Testa JRG Fukuda Y. Estudo da Paralisia Facial traumática: análise de casos clínicos e cirúrgicos Rev. Bras Otorrinolaringol. 2004; 70(4): 479-82.

16. Andrade Filho EF, Fadul Jr R, Azevedo RAA, Rocha MAD, Santos RA, Toledo SR, et al. Fraturas de mandíbula: análise de 166 casos. Rev Assoc Med Bras. 2000;46 (3):272-6.

17. Bianchini, EMG, Mangilli LD, Marzotto SR, Nazário D. Pacientes acometidos por trauma da face: caracterização, aplicabilidade e resultados do tratamento fonoaudiológico específico. Rev CEFAC. 2004; 6 (4): 388-95.

18. Lazarini PR, Fernandes AME; Brasileiro VSB, Cústódio SEV. Paralisia facial periférica por acometimento do tronco cerebral - a propósito de um caso clínico. Rev. Bras Otorrinolaringol. 2002; 68 (1): 140-4.

19. Tessitore A, Pfelsticker LN, Paschoal JR. Aspectos neurofisiológicos da musculatura facial visando a reabilitação na paralisia facial. Rev. CEFAC. 2008;10 (1): 68-75.

20. Freitas KCS, Goffi Goméz MV. Grau de percepção e incômodo quanto à condição facial em indivíduos com paralisia facial periférica na fase de seqüelas. Rev Soc Bras Fonoaudiol. 2008;13(2):113-8.

21. Rintchev A, Wernig A. Denervation and reinervation of muscle: physiological effect. Eur. Arch. Otohinolaryngol. (Suppl.) 1994; 528-30.

22. Bogar P, Calda Neto SS, Marone SAM, Miniti A. Espasmo hemifacial: Dez anos de experiência. Rev. Bras de Otorrinolaringologia. 1991; 57(1): 105-9.

23. Bernardes DFF, Goffi-Gomez MVS, Pirana $S$, Bento RF. Perfil funcional nos indivíduos com paralisia facial tratados em uma abordagem miofuncional. Pró-Fono. 2004; 16(2):151-8.

24. Rahal A, Pierotti S. Eletromiografia e cefalometria na fonoaudiologia. In: Ferreira LP, Befi-Lopes DM, Limongi SCO (organizadores). Tratado de fonoaudiologia. São Paulo: roca, 2004: 237-53.

25. Bernardes DFF, Goffi Goméz MV, Bento RF. Eletromiografia de superfície em pacientes portadores de paralisia facial periférica. Rev. CEFAC. 2010; 12(1): 91-6.
http://dx.doi.org/10.1590/S1516-18462012005000005

RECEBIDO EM: 28/01/2011

ACEITO EM: 23/08/2011

Endereço para correspondência:

Leila Bonfim de Jesus

Alameda paradiso, no 75, Pituba

Salvador - Bahia

CEP: 41830-620

E-mail: leu_fono@yahoo.com.br 